\title{
Is African-American girls' perception of their mother-daughter relationship associated to psychosocial and physical activity variables?
}

\author{
Sarah Burkart, Brett Winner, Cory Greever, Sofiya Alhassan, PhD, FACSM, University of \\ Massachusetts Amherst, Amherst, MA
}

Maternal influence has been reported to play a significant role in the health behaviors of children. In Caucasian girls, it has been reported that mother-daughter relationship can influence psychosocial variables such as physical activity (PA) self-efficacy. Currently, there is very little data on the impact of African-American girls' perception of their relationship with their mother and psychosocial variables. PURPOSE: To examine the association between motherdaughter relationship, PA self-efficacy (PA-SE) and PA levels in African-American girls.

METHODS: Baseline data from mothers ( $n=28$; age $=37.0 \pm 6.7$ years; $B M l=33.5 \pm 10.6 \mathrm{~kg} / \mathrm{m}^{2}$ ) and daughters $\left(n=32\right.$; age $=9.0 \pm 1.2$ years; $B M I=20.4 \pm 5.7 \mathrm{~kg} / \mathrm{m}^{2}, 90^{\text {th }}$ percentile) participating in an afterschool mother-daughter PA study was used in this analysis. PA was measured for 7 continuous days using accelerometers. Parental Responsiveness (PR) and Adolescent Openness to Parental Socialization scales were used to assess mother-daughter relationship. Daughters' self-esteem and depressive symptoms were assessed with the Rosenberg SelfEsteem (RSE) scale and the Child Depression Inventory (CDI), respectively. Participants' PASE was assessed with validated questionnaires. Spearman correlations were used to examine associations between mother-daughter relationship, psychosocial variables, and PA levels. Daughters' scores for the PR scale were divided into tertiles. Between group (high versus low perception of mother-daughter relationship) differences in psychosocial variables and PA were assessed with $t$-tests. RESULTS: Daughters' perception of their mother-daughter relationship was positively correlated with RSE $(r=0.36, p=0.04)$. Daughters' percent time spent in MVPA was negatively correlated with CDI $(r=-0.42, p=0.03)$ and positively correlated with mothers' PASE $(r=0.44, p=0.04)$. With respect to daughters' PR scale, significant differences in RSE score $(\mathrm{HIGH}=23.82 \pm 4.33 ; \mathrm{LOW}=18.20 \pm 4.87, p=0.01)$ and percent time spent in sedentary activity $(\mathrm{HIGH}=27.83 \pm 32.31 ; \mathrm{LOW}=57.41 \pm 20.93, p=0.02)$ were observed between tertiles.

CONCLUSION: African-American girls' perception of their relationship with their mother seems to be associated with self-esteem, depressive symptoms and sedentary pursuits. Future studies should target interventions that improve mother-daughter relationship.

Contact Information:

Sarah Burkart

(908) 910-3027

sburkart@kin.umass.edu

30 Eastman Lane

110 Totman Building

Amherst, MA 01003 